\title{
Estimates of Victim Surface Area and Associated Extraction Force for Partial Entrapment in Grain
}

\author{
C. V. Schwab, L. E. Schwab, P. J. Schwab
}

Research

\section{HighLIGHTS}

- Eight selected anthropometric landmarks were useful for estimating victim surface area and entrapment depth.

- Surface area estimates for a partially entrapped male victim ranged from 0.0716 to $2.7296 \mathrm{~m}^{2}$.

- Partial extraction force estimates for a male victim ranged from 0.29 to $3,693 \mathrm{~N}$.

- Partial extraction force estimates were $18 \%$ greater on average when including the arm surface area than when not including the arms.

ABSTRACT. A prediction model for estimating extraction forces on entrapped victims was enhanced and modernized in 2018 from the original 1985 model. The prediction model was divided into two conditions based on the victim's relative position to the grain surface. The first condition was when the victim is completely below the grain surface. The second condition was when the victim's shoulders are above the grain surface; this condition is the focus of this research. A variable in the prediction model that changes with the depth of entrapment is the surface area of the victim. A sample of 60 male models was used to approximate the human surface area at optimal discrete positions selected based on visually identifiable anthropometric landmarks. The surface area estimates for those 60 partially entrapped male models ranged from 0.0716 to $2.7296 \mathrm{~m}^{2}$. Extraction forces for twelve partially entrapped male body types with various combinations of stature and body mass index were calculated. The extraction forces were calculated for conditions when the victim's arms were raised (above the grain) and lowered (in the grain). Results from the prediction model showed that surface area contributed less to the partial extraction force for short underweight bodies than for tall extremely obese bodies. At the lower landmarks, i.e., medial malleolus (MM) and knee crease (KN), surface area did not contribute noticeably to the partial extraction force. The contribution of surface area was not noticeable until the victim was buried up to the crotch (landmark $C R$ ).

Keywords. Farm safety, Grain entrapment, Prediction model, Rescue, Safety.

\footnotetext{
(c) (1) $\odot$ The authors have paid for open access for this article. This work is licensed under a Creative Commons Attribution-NonCommercial-NoDerivatives 4.0 International License https://creative commons.org/licenses/by-nc-nd/4.0/Submitted for review on 29 July 2020 as manuscript number JASH 14230; approved for publication as a Research Article by the Ergonomics, Safety, \& Health Community of ASABE on 24 November 2020.

The authors are Charles V. Schwab, Professor, Department of Agricultural and Biosystems Engineering, and Lauren E. Schwab, Lecturer, Department of Civil, Construction, and Environmental Engineering, Iowa State University, Ames, Iowa; Pamela J. Schwab, Director, Civil Software Design LLC, Ames, Iowa. Corresponding author: Charles V. Schwab, 3335 Elings Hall, 605 Bissell Rd., Ames, IA 50011-3270; phone: 515-294-4131; email: cvschwab@iastate.edu.
}

Journal of Agricultural Safety and Health

27(1): 53-66 C 2021 ASABE ISSN 1074-7583 https://doi.org/10.13031/jash.14230 

confined spaces. Cheng et al. (2019) reported an increase of 13\% in documented confined-space injury cases in 2018 over 2017. Cheng et al. (2019) also reported that the states of Iowa, Illinois, Nebraska, Ohio, and Wisconsin had the most fatal and nonfatal confined-space cases for agriculture in 2018. The type of agricultural confined-space incident accounting for most of these documented cases is entrapment in grain, which accounts for $49 \%$ of total cases (Cheng et al., 2019). Issa et al. (2016) reported a very strong correlation between states that have more farms with grain storage capacity and those with more confined-space incidents over a ten-year period.

An improved mathematical prediction model could advance the exploration of innovative techniques to reduce the extraction forces on grain entrapment victims and increase the likelihood of survival of future entrapment victims. Better understanding of the surface area of male victims with different body types and the magnitude of the extraction forces required to extricate male victims partially trapped in grain would be useful. The purpose of this research was to reintroduce the prediction model for partially entrapped victims, explore the relationship of the victim's surface area to easily identifiable human landmarks, and produce partial extraction force estimates for male victims with different body types who are partially submerged below the grain surface.

\section{Original Prediction Model}

Schwab et al. (1985) presented the original prediction model for calculating the vertical pull required to extract a victim entrapped in grain. Estimating the extraction force requires combining the Janssen (1895) equation for predicting granular pressures, the principle of boundary shear identified by Cowin and Trent (1980), specific variables associated with the trapped victim and the granular material, and the diameter of the bin. The derivation of the original prediction model was initially presented by Schwab (1982). The original prediction model is divided into two conditions based on the victim's relative position to the grain surface. The first condition is when the victim is completely below the grain surface; the extraction force for the first condition is calculated using equation 1 :

$$
\begin{aligned}
F_{\text {extraction }}= & g \bar{W}+\frac{g w R A}{\mu k}\left(1-\exp \left(\frac{-k \mu y_{2}}{R}\right)\right) \\
& +\frac{g S \phi w R}{\mu y_{1}}\left[y_{3}-y_{2}+\frac{R}{k \mu}\left(\exp \left(\frac{-k \mu y_{3}}{R}\right)-\exp \left(\frac{-k \mu y_{2}}{R}\right)\right)\right]
\end{aligned}
$$

where

$F_{\text {extraction }}=$ force required to extract victim from grain (N)

$\bar{W}=$ mass of victim $(\mathrm{kg})$

$g=$ standard acceleration of gravity $\left(\mathrm{m} \mathrm{s}^{-2}\right)$

$w=$ bulk weight of grain $\left(\mathrm{kg} \mathrm{m}^{-3}\right)$

$R=$ hydraulic radius of cylindrical bin (m)

$A=$ top surface area of victim $\left(\mathrm{m}^{2}\right)$

$\mu=$ coefficient of friction of grain on grain (dimensionless)

$k=$ ratio of vertical to lateral pressure (dimensionless)

$S=$ surface area of victim $\left(\mathrm{m}^{2}\right)$ 
$\phi=$ coefficient of friction of grain on victim's surface (dimensionless)

$y_{1}=$ distance from top of victim's head to bottom of feet (m)

$y_{2}=$ distance from top of victim's head to top surface of grain $(\mathrm{m})$

$y_{3}=$ distance from bottom of victim's feet to top surface of grain (m).

The second condition is when the victim's shoulders are above the grain surface; the extraction force for the second condition is calculated using equation 2 :

$$
F_{\text {extraction }}=g \bar{W}+\frac{g S \phi w R}{\mu y_{1}}\left[y_{3}+\frac{R}{k \mu}\left(\exp \left(\frac{-k \mu y_{3}}{R}\right)-1\right)\right]
$$

The vertical distances used for second condition are shown in figure 1. The primary interest for this research was the second condition, with emphasis on determining the surface area of the victim below the grain and estimating the extraction force on a partially entrapped victim.

These equations assume free-flowing grain and enveloping flow conditions in a cylindrical bin with a grain height to bin diameter ratio of 1.5 or less. Grain height to bin diameter ratios of 2.0 or larger do not create an enveloping flow pattern on the top surface (i.e., inverted conical surfaces, as shown in fig. 1). The potential for submersion is greatly reduced without enveloping flow during emptying of the bin. These equations also are not intended for a victim submerged from a collapsed grain bridge, where the grain behaves differently and is not free-flowing.

For the purpose of this research, the original prediction model for calculating the

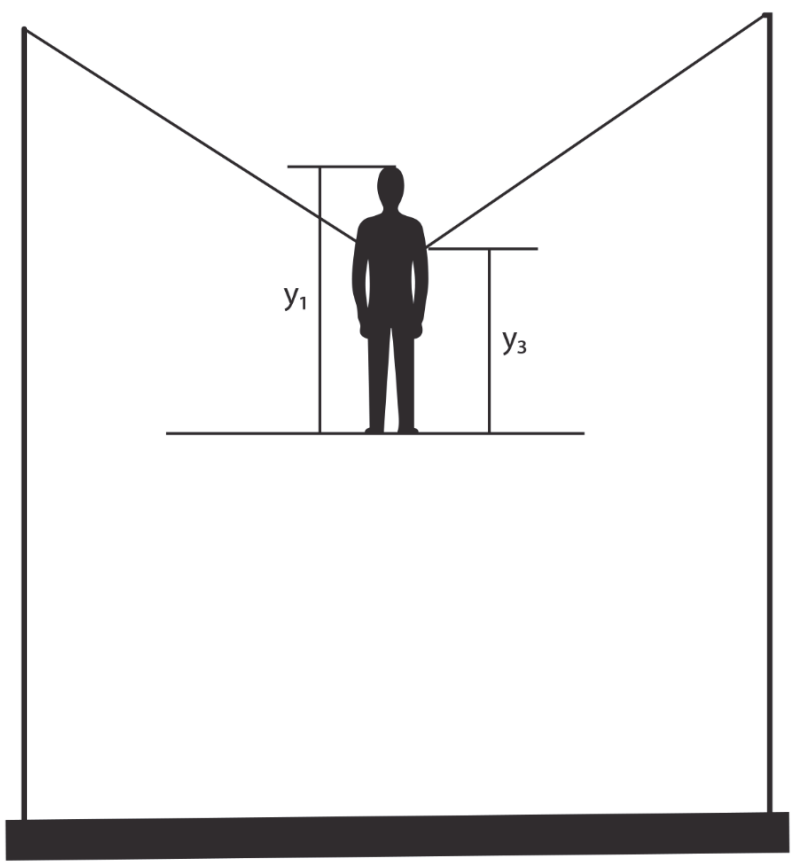

Figure 1. Two vertical distance measurements $\left(y_{1}\right.$ and $\left.y_{3}\right)$ in relation to the victim used for estimating extraction force in the second condition. 
extraction force on a partially entrapped victim was slightly modified for convenience in reporting results. The mass of the victim does not significantly influence other components of the extraction force equation. The influences caused by variations in the victim's mass are managed through the influences of stature and body mass index (BMI) on the victim's surface area. The variations in surface area are not related to minor variations of 4.4, 8.8, or $22.1 \mathrm{~kg}(2,4$, or $10 \mathrm{lb})$ in the victim's mass as much as they are to the victim being underweight or overweight based on BMI. The extraction force prediction model contains two terms, including the mass of the victim multiplied by acceleration and the partial extraction force, as shown in equation 3:

$$
F_{\text {extraction }}=g \bar{W}+F_{\text {partial extraction }}
$$

The second term in equation $3\left(F_{\text {partial extraction }}\right)$ is the partial extraction force, which is dependent on the victim's surface area and is used to express how different body types and depths of entrapment influence the estimated extraction force. The partial extraction force is calculated using equation 4 :

$$
F_{\text {partial extraction }}=\frac{g S \phi w R}{\mu y_{1}}\left[y_{3}+\frac{R}{k \mu}\left(\exp \left(\frac{-k \mu y_{3}}{R}\right)-1\right)\right]
$$

\section{Anthropometric Data}

A necessary variable for calculating the extraction force on an entrapped victim is the surface area of the victim below the grain. The frictional force developed by the grain on the victim is the product of the normal force (defined as the lateral grain pressure), the coefficient of friction of the grain on the victim's surface, and the victim's surface area below the grain surface. Human male models were used for determining surface areas. Anthropometric data from the U.S. Department of Health and Human Services (DHHS, 2016) and the human model from the University of Michigan Transportation Research Institute (UMTRI, 2018) were used to construct a computer-generated male model for determining values for a victim's surface area. A total of 60 male models were constructed using three statures (tall, medium, and short, as shown in fig. 2), four BMI values (underweight, normal, overweight, and extremely obese, as shown in fig. 3), and five ages (20, 30, 40, 50 , and 60 years). The possible body conditions were tall underweight (TU), tall normal (TN), tall overweight (TO), tall extremely obese (TE), medium underweight (MU), medium normal (MN), medium overweight (MO), medium extremely obese (ME), short underweight (SU), short normal (SN), short overweight (SO), and short extremely obese (SE).

\section{Determining the Surface Area of Partially Entrapped Victims}

The male models and computer technology allow estimating the submerged surface area of a grain entrapment victim at every millimeter of height. While this level of precision is possible, there is little practical application for millimeter-level accuracy in this research or in actual entrapment incidents. The ability to accurately determine if a victim is buried 584 or $585 \mathrm{~mm}$ deep in grain does not exist in situ. However, using a set of common human physical markers that are easy to identify from a distance has practical application for determining the surface area below the grain of a partially entrapped victim.

Eight physical markers were identified from available landmark data of human subjects. 


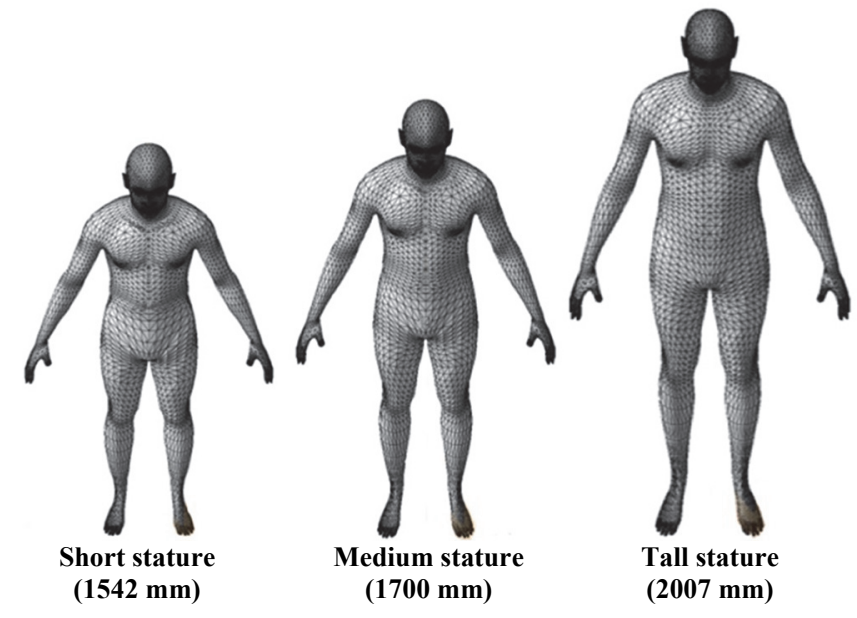

Figure 2. UMTRI computer-generated male body models for three statures with normal BMI.

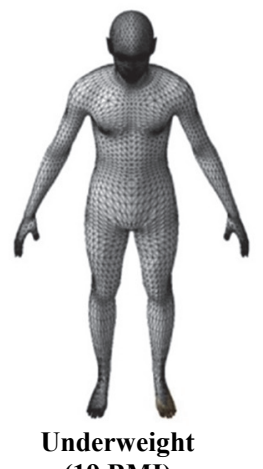

(19 BMI)

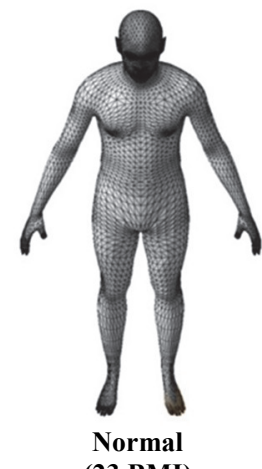

(23 BMI)

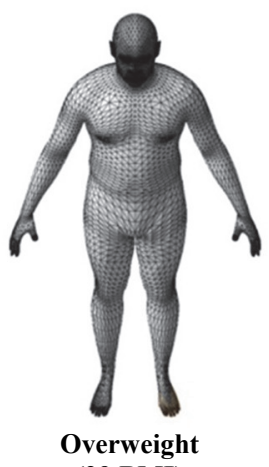

(29 BMI)

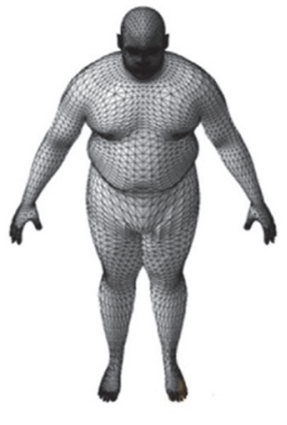

Extremely obese (40 BMI)

Figure 3. UMTRI computer-generated male body models for four BMI values with medium stature.

These landmarks are the medial malleolus (MM), knee crease (KN), crotch (CR), anterior superior iliac spine (AS), thelion (TH), acromion (AC), infraorbitale (IN), and stature (ST). These are well established and commonly accepted terms (Azouz et al., 2006). These landmarks, along with descriptions and dimensions for a tall underweight 20 -year-old male, are presented in table 1. Figure 4 shows a comparison between the landmarks for two 20 -yearold males: one tall and underweight and the other short and extremely obese.

Some of the landmarks have right and left measurements associated with specific human anatomy (e.g., right and left medial malleolus). For simplicity, an assumption was made that the right side measurement would yield a satisfactory height estimate for calculating surface area. Variations between right and left side measurements can exist but are considered negligible in this research.

Each male model was sliced at the heights indicated by the landmarks, and surface area calculations were performed for the portion of the male model that was submerged up to each landmark. Surface area measurements were recorded for all combinations of the three 
Table 1. Human landmark identifiers and values for a tall underweight 20-year-old male.

\begin{tabular}{|c|c|c|c|c|}
\hline Code & Landmark Definition & Description & $\begin{array}{l}\operatorname{Dimension}^{[a]} \\
(\mathrm{mm})\end{array}$ & $\begin{array}{c}\text { Percentage } \\
\text { of Height }^{[b]} \\
(\%)\end{array}$ \\
\hline MM & Right and left medial malleolus & Ankle height & 97.9 & 5 \\
\hline $\mathrm{KN}$ & Right and left knee crease & Knee height & 584.8 & 29 \\
\hline $\mathrm{CR}$ & Crotch & Crotch height & 963.5 & 48 \\
\hline AS & $\begin{array}{l}\text { Right and left anterior superior } \\
\text { iliac spine (ASIS) }\end{array}$ & Waist height & 1172.1 & 58 \\
\hline $\mathrm{TH}$ & Right and left thelion & Bust height & 1484.1 & 74 \\
\hline $\mathrm{AC}$ & Right and left acromion & Shoulder height & 1667.0 & 83 \\
\hline IN & Right and left infraorbitale & Eye height & 1863.7 & 93 \\
\hline ST & Stature & $\begin{array}{l}\text { Height from bottom of } \\
\text { feet to top of head }\end{array}$ & 2007.0 & 100 \\
\hline
\end{tabular}

[a] Distance in millimeters from bottom of feet to the location of the landmark.

[b] Calculated by dividing the dimension for each landmark by the height of the male sample.

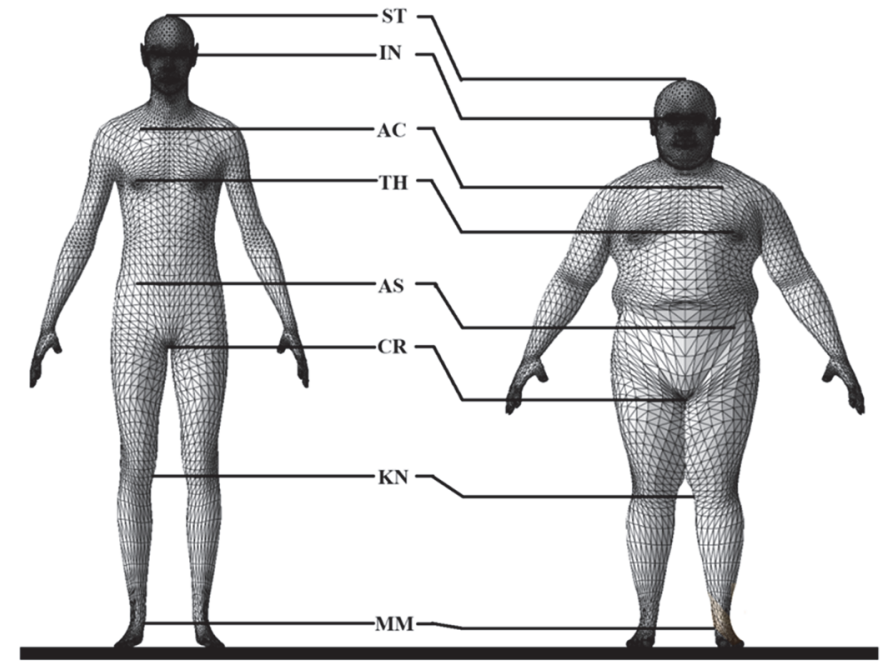

Figure 4. Landmark locations on two 20-year-old males: tall underweight and short extremely obese.

statures, four body types, five ages, and eight landmark locations, yielding 480 human model records. The surface areas were also measured using two conditions related to the victim's arms. One surface area measurement included the contribution of the victim's arms below the surface of the grain. The other surface area measurement excluded the contribution of the surface area of the victim's arms. From a practical perspective, a partially submerged victim may have both arms extended overhead, e.g., to hold on to a rope or grasp for something to aid in extraction. In those cases, the victim's arms should not be added to the victim's surface area below the grain surface. The opposite is true when the victim's arms are below the grain surface. In that case, the surface area contribution from the arms must be included.

\section{Surface Areas of Partially Entrapped Victims}

The surface areas for partially entrapped male victims ranged from $0.0716 \mathrm{~m}^{2}$ at landmark MM on the SU victim to $2.7296 \mathrm{~m}^{2}$ at landmark ST on the TE victim. Landmarks 
$\mathrm{MM}$ and $\mathrm{KN}$ showed a very tight grouping of results and surface areas that were less than $22 \%$ of the total surface area (fig. 5). Similarly, landmarks IN and ST showed a tight grouping of results and less than $10 \%$ of the total surface area. Most of the surface area (68\%) accumulated at four landmarks: $\mathrm{CR}, \mathrm{AS}, \mathrm{TH}$, and AC. At these four landmarks, there were more variations in the percentages of height and surface area, as shown by the scattering of the data points on both the horizontal and vertical axes.

A statistical analysis was used to determine if the independent variables for stature ( $\mathrm{T}$, $\mathrm{M}$, and S), body type (U, N, O, and E), age (20, 30, 40, 50, and 60), and landmark (MM, $\mathrm{KN}, \mathrm{CR}, \mathrm{AS}, \mathrm{TH}, \mathrm{AC}, \mathrm{IN}$, and ST) had impacts on the dependent variable, i.e., the male victim's measured surface area with arms. Stature, body type, and landmark were shown to have statistical probability of influencing the victim's measured surface area with arms. Results also showed that age had no significant relationship with measured surface area with arms, and age was therefore not a necessary parameter for estimating the extraction force on an entrapped victim. This result agrees with the findings of Schwab et al. (2019), who determined that the age of a human model had no significant relationship with surface area for a completely submerged victim. The values for the victim's surface area with arms were pooled for the age values.

A similar statistical analysis was used to determine if the independent variables had impacts on the male victim's measured surface area without arms. It was determined that stature, body type, and landmark had statistical probability of influencing the victim's measured surface area. Again, age had no significant relationship with the measured surface area without arms. The values for the victim's surface area without arms were pooled for the age values.

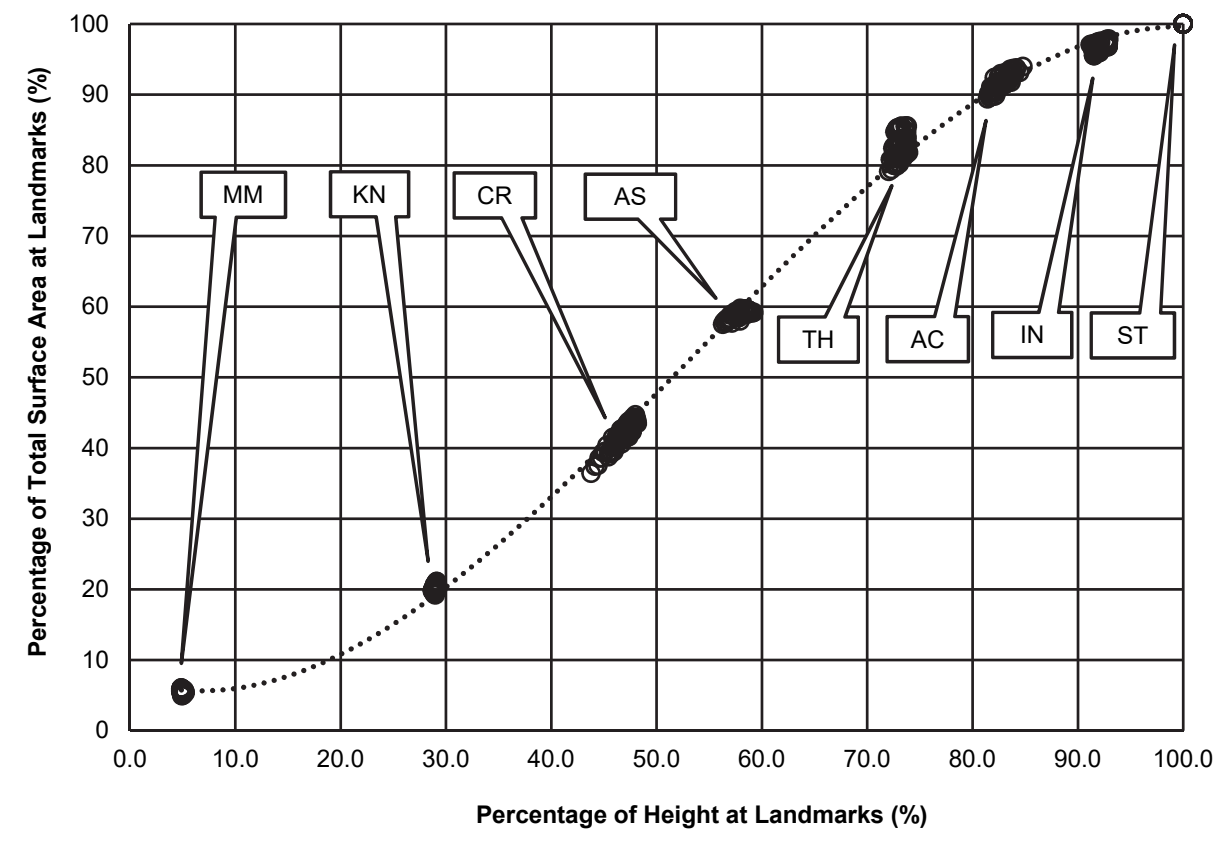

Figure 5. Plot of all data points for percentages of surface area versus height at eight landmarks. 


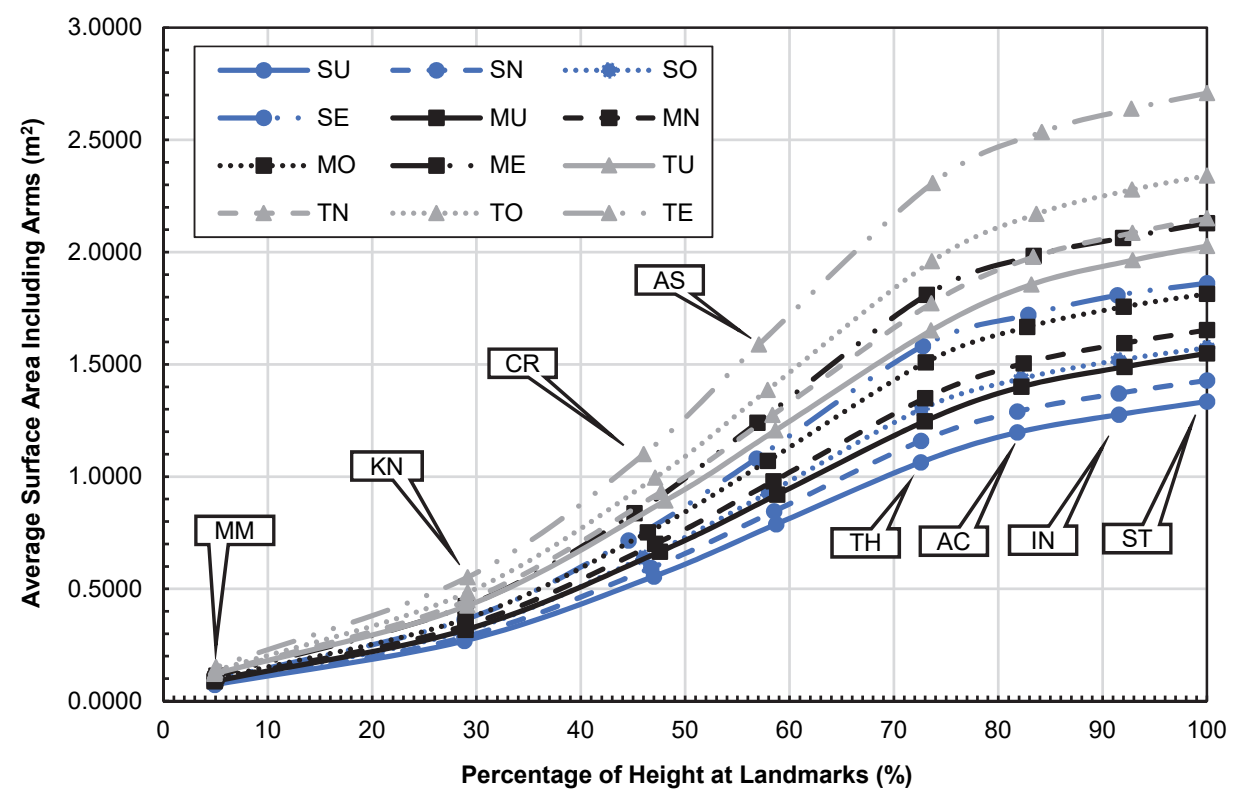

Figure 6. Average surface areas of male victim, including arms, at eight landmarks for all combinations of three statures and four body types.

The variations in the average measured surface area of a male victim, including the arms, for the eight landmarks by stature and body type are shown in figure 6, which illustrates the small amount of variation at landmark MM versus the large variation at landmark ST. The short victim's average surface area values overlap somewhat with the medium victim's average surface area values, while the medium victim's average surface area values overlap somewhat with the tall victim's average surface area values.

The variations in the average measured surface area of a male victim, not including the arms, for the eight landmarks by stature and body type are shown in figure 7. The minimum measured surface area was the same without arms as with arms because the victim's arms are not below the grain surface for landmarks $\mathrm{MM}$ and $\mathrm{KN}$, where the minimum values are located. Therefore, the inclusion of arms has no impact on the minimum measured values. The maximum measured surface area without arms was $2.3036 \mathrm{~m}^{2}$, which is $0.4260 \mathrm{~m}^{2}$ smaller than the maximum measured surface area with arms. Similar overlaps exist between different statures for the average measured surface area with arms and without arms.

The male victim's average surface area with and without arms exhibited variations influenced by the independent variables for stature (T, M, S), body type (U, N, O, E), and landmark (MM, KN, CR, AS, TH, AC, IN, ST). These variations are visible in figures 6 and 7, but the importance of these variations can best be understood in their influence on the extraction force necessary for a partially entrapped male victim.

\section{Extraction Force on Partially Entrapped Victim}

The partial extraction force was estimated using equation 4 with the different depths of entrapment based on the landmarks $\left(y_{3}\right)$, victim stature $\left(y_{1}\right)$, and surface area $(S)$, which were dependent on the victim's body type. The ranges of $y_{1}, y_{3}, S$, and the remaining fixed 


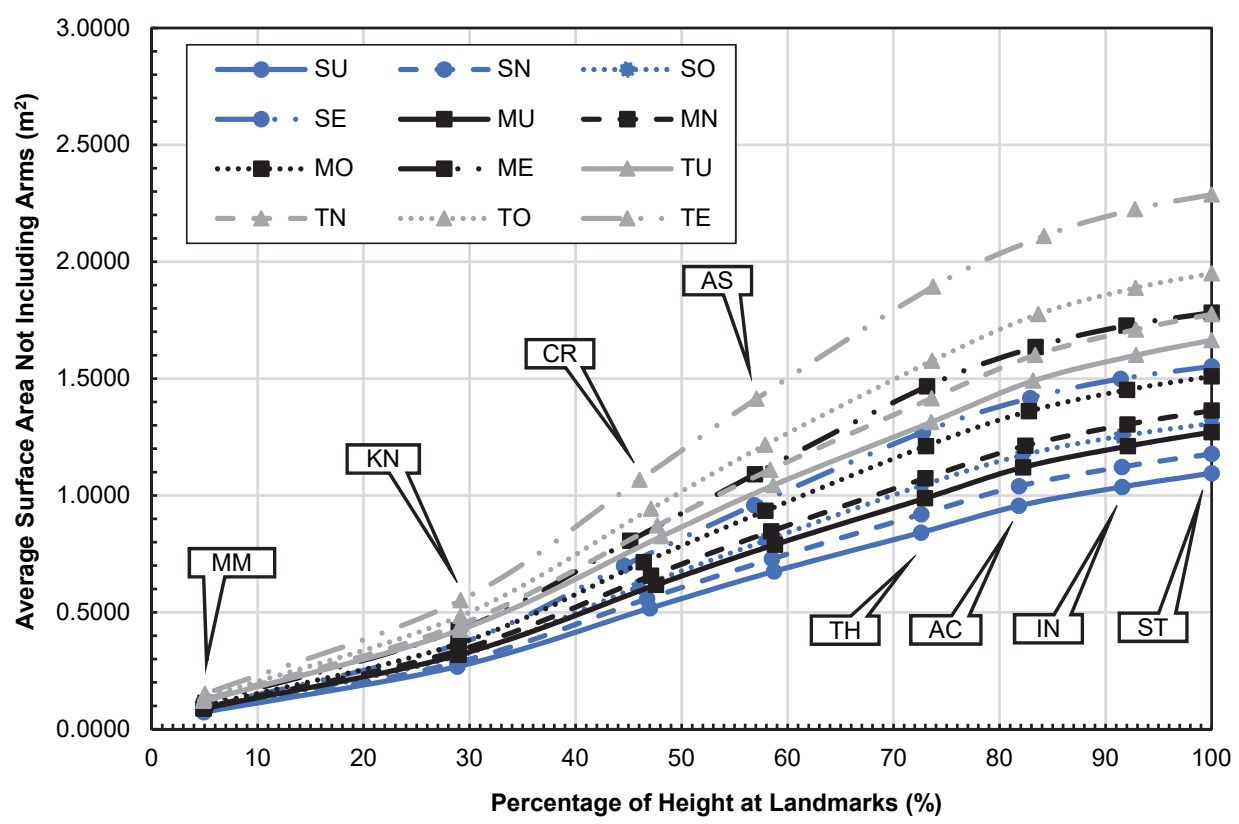

Figure 7. Average surface areas of male victim, not including arms, at eight landmarks for all combinations of three statures and four body types.

Table 2. Independent variables for partial extraction force prediction model and ranges for variables that were allowed to change.

\begin{tabular}{cccc}
\hline & & \multicolumn{2}{c}{ Base Values } \\
\cline { 3 - 4 } Independent Variables & & SI Units & U.S. Units \\
\hline Fixed variables & $\mu$ & $0.38 \mathrm{dl}^{[\mathrm{a}]}$ & $0.38 \mathrm{dl}$ \\
& $R$ & $2.3 \mathrm{~m}^{[\mathrm{b}]}$ & $7.5 \mathrm{ft}$ \\
& $w$ & $730 \mathrm{~kg} \mathrm{~m}^{-3}[\mathrm{c}]$ & $45.6 \mathrm{lb} \mathrm{ft}^{-3}$ \\
& $\phi$ & $0.6 \mathrm{dl}{ }^{[\mathrm{d}]}$ & $0.6 \mathrm{dl}$ \\
& $k$ & $0.5 \mathrm{dl}^{[\mathrm{e}]}$ & $0.5 \mathrm{dl}$ \\
\hline Ranging variables & $S$ & 0.0728 to $2.7082 \mathrm{~m}^{2}$ & 0.784 to $29.151 \mathrm{ft}^{2}$ \\
& $y_{1}$ & 1.542 to $2.007 \mathrm{~m}$ & 5.06 to $6.58 \mathrm{ft}^{2}$ \\
& $y_{3}$ & 0.0758 to $2.007 \mathrm{~m}$ & 0.25 to $6.58 \mathrm{ft}$ \\
\hline
\end{tabular}

[a] The coefficient of friction used was for corn on structural corrugated steel surfaces (Schwab et al., 1985).

[b] The hydraulic radius of the bin is for a $9.144 \mathrm{~m} \mathrm{(30} \mathrm{ft)} \mathrm{diameter} \mathrm{bin.}$

[c] The bulk density of grain is for corn, experimentally determined from samples (Schwab et al., 1985).

[d] The average apparent coefficient of friction of corn on male victim's surface (Schwab et al., 1985).

[e] The ratio of lateral to vertical pressure is from ASABE Standard EP433 (ASABE, 2016).

variables are provided in table 2. Note that these estimated partial extraction forces do not contain the force contributed by the victim's mass multiplied by the gravitational constant. Therefore, the partial extraction force estimates must be added to the force contributed by the victim's mass before comparing these extraction force results with other research.

The partial extraction force estimates for all statures and body types by percentage of height at the landmarks are shown in figure 8 . The partial extraction force estimates, including the surface area of the victim's submerged arms, ranged from a minimum of 0.29 


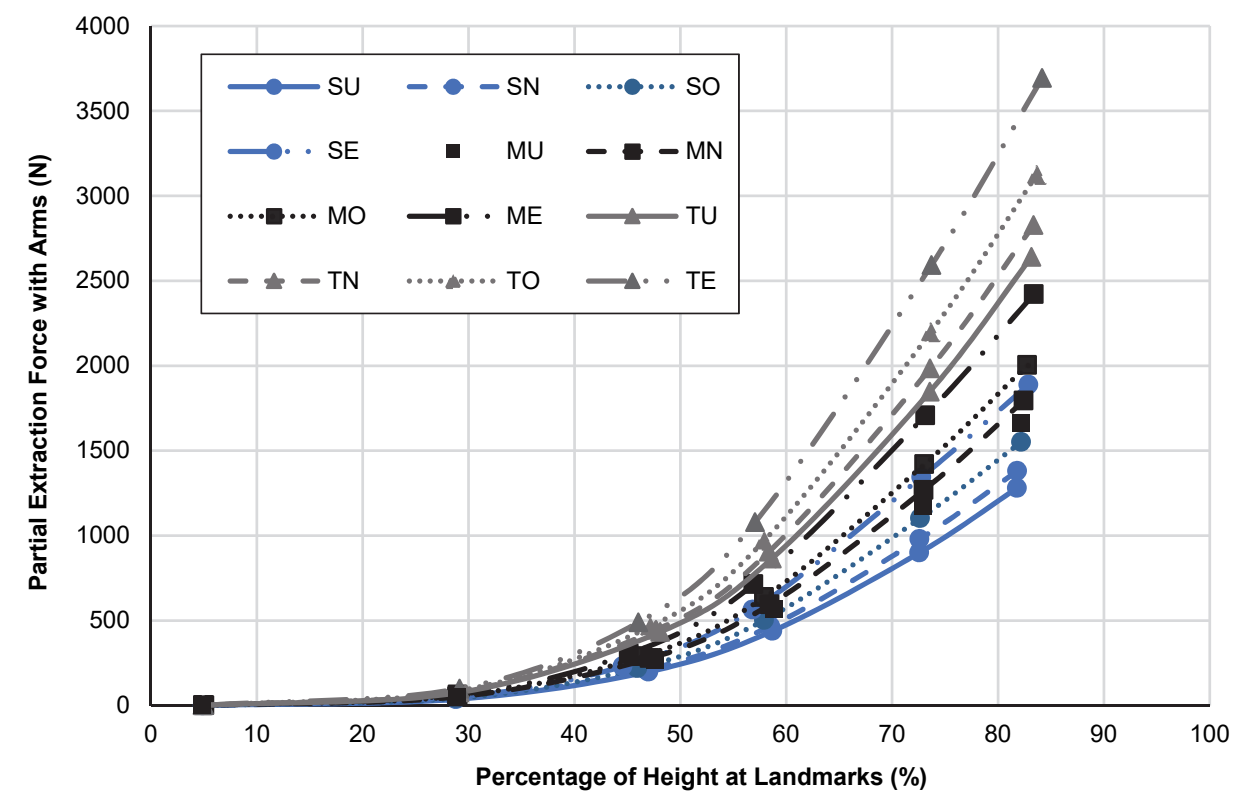

Figure 8. Partial extraction force for male victim, including arms, by percentage of height at landmarks for all combinations of three statures and four body types.

$\mathrm{N}(0.065 \mathrm{lbf})$ at landmark MM for a male of SU body type to a maximum of 3,693 N (830 $\mathrm{lbf}$ ) at landmark AC for a male of TE body type. The contribution of the partial extraction force to the extraction force value was less than $10 \%$ until the depth associated with landmark CR was reached. This was consistent with the finding that $68 \%$ of the human surface area is located between CR and AC.

Figure 9 is a scaled representation of the partial extraction force for male victims of different stature and body type when buried up to landmark TH. The average surface area and force estimates for the different statures, BMI values, and landmarks are presented in tables 3, 4, and 5. These tables assist in identifying when the partial extraction force estimate exceeds the mass contribution estimate.

The partial extraction force estimates, excluding the surface area of the arms, ranged from a minimum of $0.29 \mathrm{~N}(0.065 \mathrm{lbf})$ for a male victim of SU body type buried to a depth of MM to a maximum of 3,074 N (691 lbf) for a male victim of TE body type buried to a depth of AC. The minimum values for partial extraction force are identical for both conditions, i.e., including and excluding the arms, because the victim's arms do not influence the extraction force at landmark MM. The surface area of the arms does not become a factor until landmark CR is reached. The partial extraction force estimates, excluding the surface area of the arms, for all statures and body types by percentage of height at the landmarks are shown in figure 10. The percentage increase in extraction force is on average $18 \% \pm 7 \%$ greater when including the arms than without the arms.

\section{Summary}

This research presented the existing extraction force prediction model for male victims 


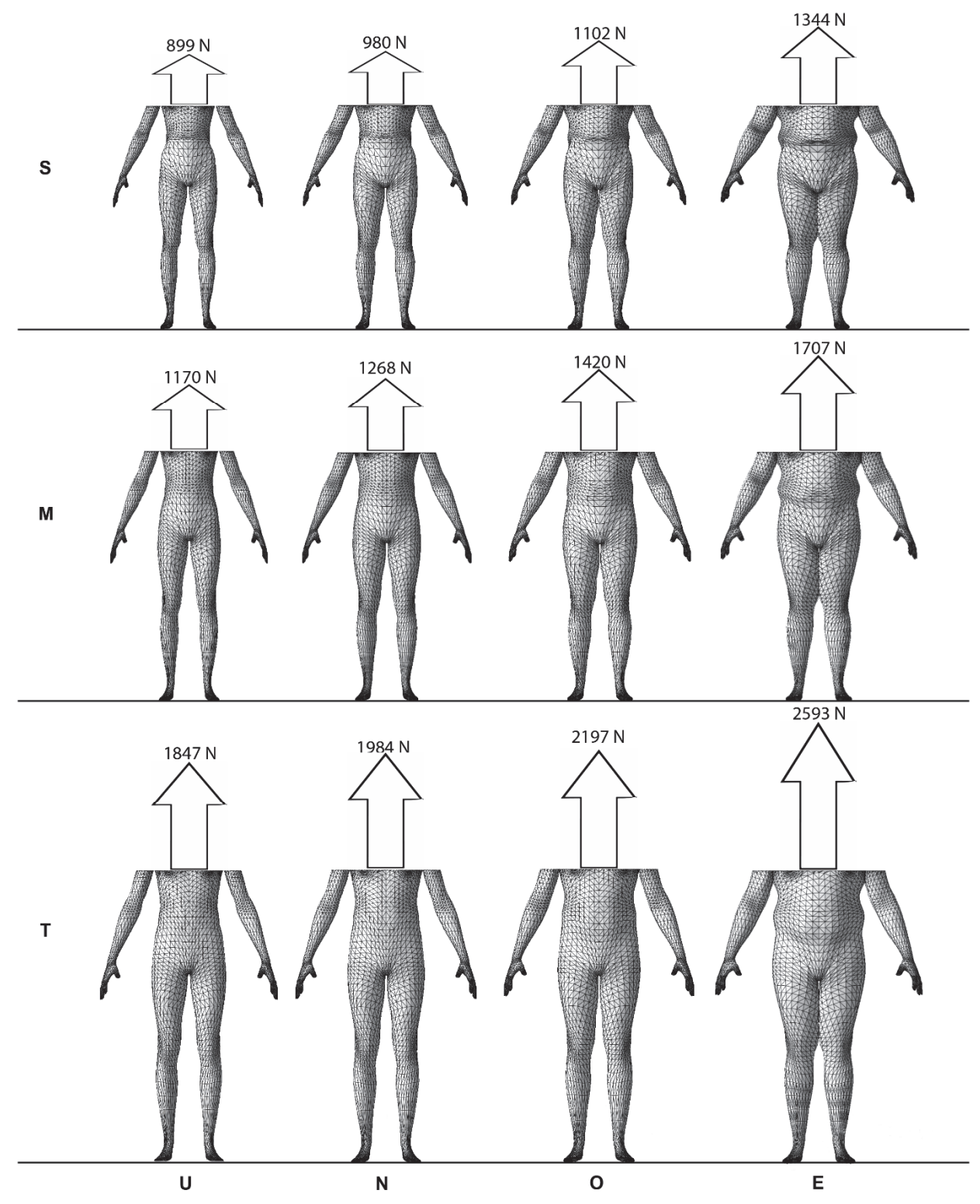

Figure 9. Graphically scaled representation of victim's body, including arms, and partial extraction force when buried to the thelion (TH) landmark for all statures and body types.

partially entrapped in grain, the surface areas for male victims of different body types who are partially entrapped, and the partial extraction force estimates for male victims of different body types who are buried in grain up to the shoulders. The eight human landmarks (MM, KN, CR, AS, TH, AC, IN, and ST) were useful for calculating the surface area and identifying the entrapment depths of victims. A total of 60 male models were used to 
Table 3. Average values of surface area (including arms) and forces for small stature male with different body mass index (BMI) values when buried to landmarks from crotch (CR) to acromion (AC).

\begin{tabular}{|c|c|c|c|c|c|}
\hline $\begin{array}{c}\text { Stature and } \\
\text { BMI }\end{array}$ & Landmark & $\begin{array}{c}\text { Surface Area } \\
\left(\mathrm{m}^{2}\right)\end{array}$ & $\begin{array}{l}\text { Partial Extraction } \\
\text { Force }^{[\mathrm{a}]} \\
(\mathrm{N}) \\
\end{array}$ & $\begin{array}{c}\text { Mass } \\
\text { Contribution }^{[\mathrm{b}]} \\
(\mathrm{N})\end{array}$ & $\begin{array}{l}\text { Extraction } \\
\text { Force }^{[\mathrm{c}]} \\
(\mathrm{N})\end{array}$ \\
\hline \multirow[t]{4}{*}{$\mathrm{SU}$} & $\mathrm{CR}$ & 0.5553 & 199 & 443 & 642 \\
\hline & AS & 0.7880 & 439 & 443 & 882 \\
\hline & TH & 1.0369 & 899 & 443 & 1,342 \\
\hline & $\mathrm{AC}$ & 1.1968 & 1,280 & 443 & 1,723 \\
\hline \multirow[t]{4}{*}{$\mathrm{SN}$} & $\mathrm{CR}$ & 0.5932 & 210 & 536 & 746 \\
\hline & AS & 0.8458 & 468 & 536 & 1,004 \\
\hline & TH & 1.1590 & 980 & 536 & 1,516 \\
\hline & $\mathrm{AC}$ & 1.2899 & 1,380 & 536 & 1,916 \\
\hline \multirow[t]{4}{*}{$\mathrm{SO}$} & $\mathrm{CR}$ & 0.6392 & 219 & 676 & 895 \\
\hline & AS & 0.9268 & 502 & 676 & 1,178 \\
\hline & TH & 1.3012 & 1,102 & 676 & 1,778 \\
\hline & $\mathrm{AC}$ & 1.4362 & 1,551 & 676 & 2,227 \\
\hline \multirow[t]{4}{*}{ SE } & $\mathrm{CR}$ & 0.7155 & 231 & 932 & 1,163 \\
\hline & AS & 1.0804 & 564 & 932 & 1,496 \\
\hline & TH & 1.5819 & 1,344 & 932 & 2,276 \\
\hline & $\mathrm{AC}$ & 1.7209 & 1,889 & 932 & 2,821 \\
\hline
\end{tabular}

[a] Calculated using equation 4.

[b] Estimated by multiplying male victim mass by gravitational constant.

[c] Calculated by combining partial extraction force and victim mass contribution, as shown in equation 3 .

Table 4. Average values of surface area (including arms) and forces for medium stature male with different body mass index (BMI) values when buried to landmarks from crotch (CR) to acromion (AC).

\begin{tabular}{cccccc}
\hline $\begin{array}{c}\text { Stature and } \\
\text { BMI }\end{array}$ & Landmark & $\begin{array}{c}\text { Surface Area } \\
\left(\mathrm{m}^{2}\right)\end{array}$ & $\begin{array}{c}\text { Partial Extraction } \\
\text { Force }^{[\mathrm{a}]} \\
(\mathrm{N})\end{array}$ & $\begin{array}{c}\text { Mass } \\
\text { Contribution } \\
(\mathrm{b}]\end{array}$ & $\begin{array}{c}\text { Extraction } \\
\text { Force }^{[\mathrm{cc}]} \\
(\mathrm{N})\end{array}$ \\
\hline MU & $\mathrm{CR}$ & 0.6657 & 269 & 538 & 807 \\
& $\mathrm{AS}$ & 0.9197 & 565 & 538 & 1,103 \\
& $\mathrm{TH}$ & 1.2467 & 1,170 & 538 & 1,708 \\
& AC & 1.4001 & 1,661 & 538 & 2,199 \\
\hline MN & CR & 0.7004 & 278 & 651 & 929 \\
& AS & 0.9796 & 594 & 651 & 1,245 \\
& TH & 1.3496 & 1,268 & 651 & 1,919 \\
& AC & 1.5943 & 1,795 & 651 & 2,446 \\
\hline MO & CR & 0.7519 & 289 & 821 & 1,110 \\
& AS & 1.0699 & 637 & 821 & 1,458 \\
& TH & 15093 & 1,420 & 821 & 2,241 \\
& AC & 1.6665 & 2,004 & 821 & 2,825 \\
\hline ME & CR & 0.8369 & 305 & 1,133 & 1,438 \\
& AS & 1.2399 & 714 & 1,133 & 1,847 \\
& TH & 1.8099 & 1,707 & 1,133 & 2,840 \\
& AC & 1.9836 & 2,421 & 1,133 & 3,554 \\
\hline
\end{tabular}

[a] Calculated using equation 4.

[b] Estimated by multiplying male victim mass by gravitational constant.

[c] Calculated by combining partial extraction force and victim mass contribution, as shown in equation 3 .

calculate the surface area, and the surface area estimates for partially entrapped male victims of different statures and body types ranged from 0.0716 to $2.7296 \mathrm{~m}^{2}$.

Partial extraction force estimates ranged from 0.29 to 3,693 $\mathrm{N}$ for a male victim buried up to the medial malleolus (MM) and acromion (AC), respectively. The results followed the expectation that surface area contributed less to the required partial extraction force for 
Table 5. Average values of surface area (including arms) and forces for tall stature male with different body mass index (BMI) values when buried to landmarks from crotch (CR) to acromion (AC).

\begin{tabular}{cccccc}
\hline $\begin{array}{c}\text { Stature and } \\
\text { BMI }\end{array}$ & Landmark & $\begin{array}{c}\text { Surface Area } \\
\left(\mathrm{m}^{2}\right)\end{array}$ & $\begin{array}{c}\text { Partial Extraction } \\
\text { Force }^{[\mathrm{a}]} \\
(\mathrm{N})\end{array}$ & $\begin{array}{c}\text { Mass } \\
\text { Contribution } \\
(\mathrm{N})\end{array}$ & $\begin{array}{c}\text { Extraction } \\
\text { Force }^{[\mathrm{c}]} \\
(\mathrm{N})\end{array}$ \\
\hline TU & CR & 0.8927 & 433 & 750 & 1,183 \\
& AS & 1.2057 & 865 & 750 & 1,615 \\
& TH & 1.6507 & 1,847 & 750 & 2,597 \\
& AC & 1.8556 & 2,641 & 750 & 3,391 \\
\hline TN & CR & 0.9322 & 445 & 908 & 1,353 \\
& AS & 1.2739 & 905 & 908 & 1,813 \\
& TH & 1.7714 & 1,984 & 908 & 2,892 \\
& AC & 1.9783 & 2,828 & 908 & 3,736 \\
\hline TO & CR & 0.9952 & 463 & 1,145 & 1,608 \\
& AS & 1.3860 & 969 & 1,145 & 2,114 \\
& TH & 1.9591 & 2,197 & 1,145 & 3,342 \\
& AC & 2.1695 & 3,122 & 1,145 & 4,267 \\
\hline TE & CR & 1.0995 & 489 & 1,579 & 2,068 \\
& AS & 1.4135 & 1,079 & 1,579 & 2,658 \\
& TH & 2.3069 & 2,593 & 1,579 & 4,172 \\
& AC & 2.5348 & 3,693 & 1,579 & 5,272 \\
\hline
\end{tabular}

[a] Calculated using equation 4 .

[b] Estimated by multiplying male victim mass by gravitational constant.

[c] Calculated by combining partial extraction force and victim mass contribution, as shown in equation 3 .

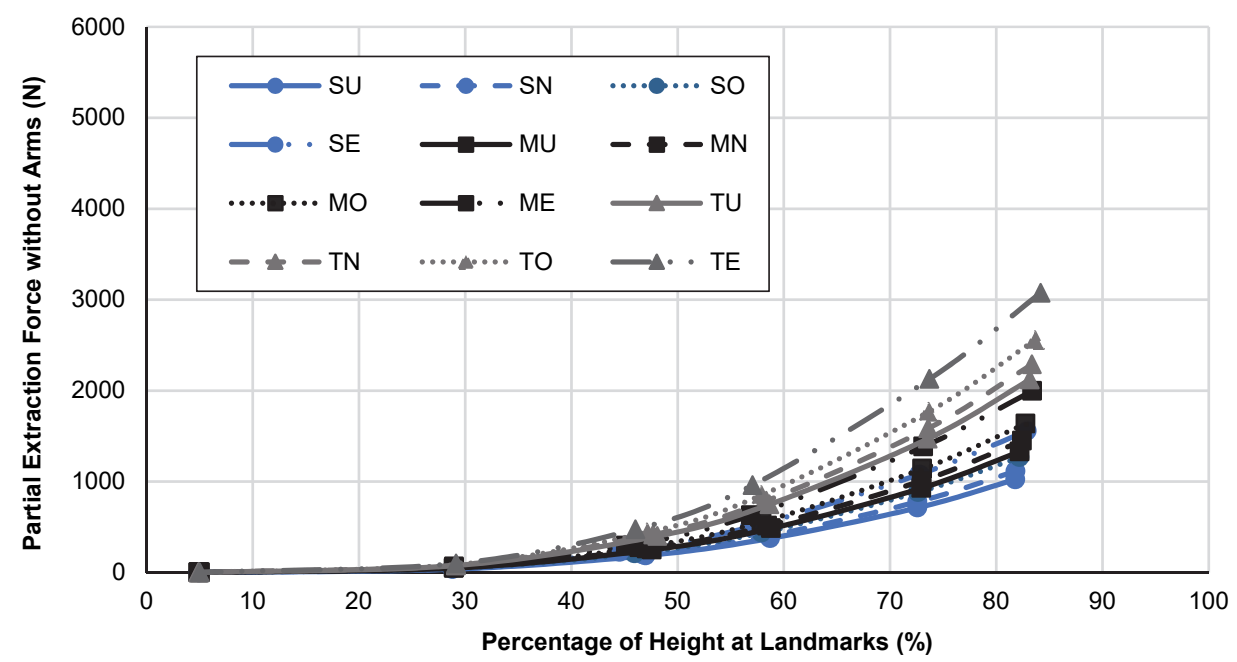

Figure 10. Partial extraction force for male victim, not including arms, by percentage of height at landmarks for all combinations of three statures and four body types.

short and underweight bodies than for tall and extremely obese bodies. The contribution of surface area did not contribute noticeably to the partial extraction force for victims buried at the lower landmarks (MM and $\mathrm{KN}$ ). The contribution of surface area became noticeable when landmark CR was reached. The partial extraction force estimates were on average $18 \%$ greater when including the surface area of the victim's arms than without the arms.

These surface area estimates and partial extraction forces for partially entrapped male 
victims of different statures and body types provide a more complete understanding of what could be expected for male victims partially buried in grain who are not $75 \mathrm{~kg}$ and 1.829 $\mathrm{m}$ tall, as specified in the original 1985 model.

\section{Acknowledgements}

Journal paper of the Iowa Agriculture and Home Economics Experiment Station, Ames, Iowa. This work was supported by the USDA National Institute of Food and Agriculture (Hatch Project No. IOW05542) and by State of Iowa funds.

\section{References}

ASABE. (2016). EP433: Loads exerted by free-flowing grain on bins. St. Joseph, MI: ASABE.

Azouz, Z. B., Shu, C., \& Mantel, A. (2006). Automatic locating of anthropometric landmarks on 3D human models. Proc. 3rd Intl. Symp. on 3D Data Processing, Visualization, and Transmission. 1, pp. 750-757. Piscataway, NJ: IEEE. https://doi.org/10.1109/3DPVT.2006.34

Cheng, Y.-H., Nour, M., Field, B., Ambrose, K., \& Sheldon, E. (2019). 2018 Summary of U.S. agricultural confined space-related injuries and fatalities. West Lafayette, IN: Purdue University, Retrieved from https://extension.entm.purdue.edu/grainsafety/grainEntrapment.php

Cowin, S. C., \& Trent, L. E. (1980). Force of extraction for a cylinder buried in sand. J. Appl. Mech., 47(4), 969-971. https://doi.org/10.1115/1.3153829

DHHS. (2016). Anthropometric reference data for children and adults: United States, 2011-2014. DHHS Publ. No. 2016-1604. Washington, DC: U.S. Department of Health and Human Services.

Issa, S. F., Cheng, Y.-H., \& Field, W. (2016). Summary of agricultural confined-space related cases: 1964-2013. J. Agric. Saf. Health, 22(1), 33-45. https://doi.org/10.13031/jash.22.10955

Janssen, H. A. (1895). Versuche über getreidedruck in silozellen. Verein Deutschen Ingenieure Zeitschrift, 39, 1045-1049.

Schwab, C. V. (1982). Inflow velocity and forces acting on a person trapped in enveloping flow of granular material. Unpublished MS thesis. Lexington, KY: University of Kentucky, Department of Agricultural Engineering.

Schwab, C. V., Schwab, L. E., \& Schwab, P. J. (2019). Extraction force prediction for male entrapment victims with different body types submerged below the grain surface. J. Agric. Saf. Health, 25(2), 77-90. https://doi.org/10.13031/jash.13155

Schwab, C. V., Ross, I. J., Piercy, L. R., \& McKenzie, B. A. (1985). Vertical pull and immersion velocity of mannequins trapped in enveloping grain flow. Trans. ASAE, 28(6), 1997-2002. https://doi.org/10.13031/2013.32555

UMTRI. (2018). Online statistical body shape models. Ann Arbor, MI: University of Michigan Transportation Research Institute. Retrieved from http://humanshape.org/ 